\title{
LINFOMA RENAL PRIMARIO EN PACIENTE CON GAMMAPATÍA MONOCLONAL IgM
}

\author{
O. RODRÍGUEZ FABA, J.M. FERNÁNDEZ GÓMEZ, J.L. MARTÍN BENITO, \\ L. PARRA MUNTANER*, A.M. GUTIÉRREZ PALACIOS**, J. GARCÍA RODRÍGUEZ, \\ A. JALÓN MONZÓN, J. REGADERA SEJAS
}

Servicio de Urología I. Hospital Central de Asturias. Facultad de Medicina. Universidad de Oviedo. Asturias. *Servicio de Urología. Hospital del Bierzo. Ponferrada. León. **Servicio de Anatomía Patológica I. Hospital Central de Asturias. Oviedo (Asturias).

Actas Urol Esp. 28 (5): 396-398, 2004

\section{RESUMEN}

LINFOMA RENAL PRIMARIO EN PACIENTE CON GAMMAPATÍA MONOCLONAL IgM

Son infrecuentes los casos de linfoma renal primario, ya que la afectación renal por un proceso linfoproliferativo es, por lo general, secundaria a una enfermedad sistémica.

Presentamos el caso de un paciente varón de 77 años que acude por hallazgo ecográfico incidental de una masa en su riñón izquierdo. Después de realizar estudios (TC), se practica nefrectomía cuyo resultado anatomopatológico fue de linfoma no-hodking B primario renal. Asimismo el paciente presentaba una gammapatía monoclonal IgM asociada, por lo que precisó tratamiento quimioterápico sistémico.

Realizamos una revisión bibliográfica centrándonos en los criterios diagnósticos y terapéuticos actuales.

PALABRAS CLAVE: Linfoma renal. Riñón. Linfoma no-hodking. IgM.

\section{ABSTRACT}

PRIMARY RENAL LYMPHOMA IN A PATIENT WITH IgM MONOCLONAL GAMMAPATHY

Reports on primary renal lymphoma are scarce in the urological literature, the most part of them are secondary on a lymphomatous infiltration of the kidneys. We report the case of a 77 year old man with an incidental mass on the kidney. After radiological studies (CT), we practise nephrectomy with a pathological result of a non-hodking B primary lymphoma. The patient present a IgM monoclonal gammapathy who need complementary treatment with chemotherapy. A literature review on currently recommended diagnostic and treatment practices in presented.

KEY WORDS: Renal lymphoma. Kidney. Non-hodgkin's lymphoma. IgM.

$\mathrm{L}$ a afectación renal por un proceso de tipo linfomatoso, se presenta en la mayor parte de los casos en el contexto de una diseminación sistémica, apreciándose adenopatías en diversos territorios y alteraciones en otros órganos.

La afectación primaria del riñón es muy rara y presenta como variedad más frecuente el tipo nohodking $^{1}$.
A continuación presentamos un nuevo caso de linfoma renal primario.

\section{CASO CLÍNICO}

En julio de 2000 y procedente del Servicio de Nefrología donde estaba siendo estudiado por una insuficiencia renal crónica secundaria a nefroangioesclerosis, nos es remitido un varón de 
77 años por el hallazgo ecográfico de una masa renal de $6 \mathrm{~cm}$ que afectaba al riñón izquierdo.

Como antecedentes personales destacables presentaba EPOC, HTA y ulcus duodenal con estenosis pilórica.

Para completar estudios realizamos un TC abdomino-pélvico, que fue informado como "lesión infiltrante que interesa los 2/3 superiores del riñón izquierdo con afectación del espacio pericapsular y bordes mal definidos" (Fig. 1). Analíticamente era muy destacable la presencia de una gammapatía monoclonal con IgM de 4640 g/l (38-231) kappa por inmunofijación. Otros análisis: proteinuria de bence-jones positiva, aspirado de médula ósea con $12,5 \%$ de atipias y serie ósea sin lesiones líticas.

Con el primer diagnóstico de masa renal asociada a gammapatía monoclonal IgM (sin cumplir criterios de mieloma IgM), se practica en agosto de 2000 nefrectomía radical izquierda.

El resultado anatomopatológico fue de linfoma no-hodking B primario renal tipo MALT (tumor linfoide asociado a las mucosas).

El paciente presenta una buena evolución post-operatoria y es dado de alta con controles ambulatorios. En diciembre de 2000 se realizó un primer control evidenciándose una IgM de 3430 $\mathrm{g} / 1$ en un primer análisis y de $4340 \mathrm{~g} / 1$ en un segundo, por lo que se decidió por parte del Servicio de Hematología comenzar tratamiento con Clorambucil. Se practicó un TC de control que fue informado como "engrosamiento de fascia perirrenal con desflecamiento de grasa compati-

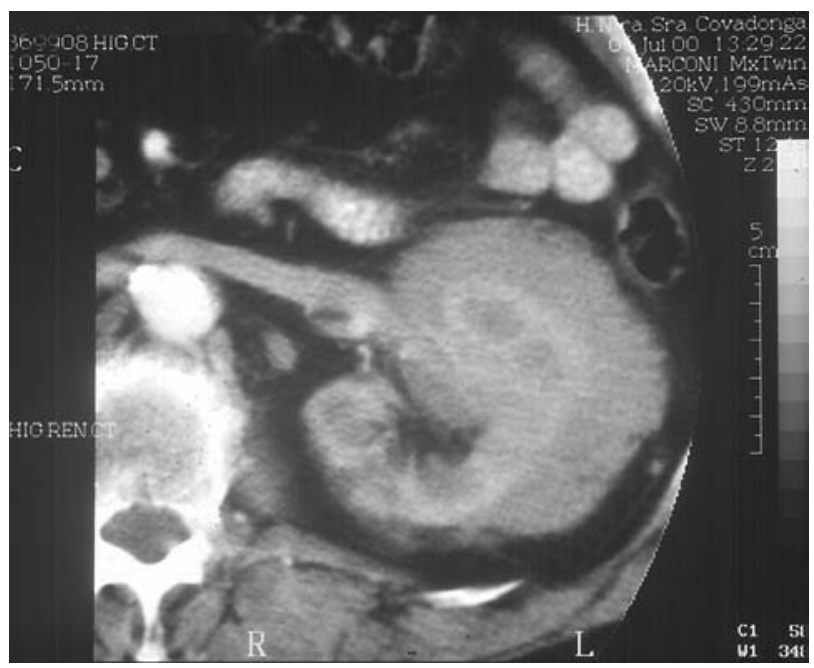

FIGURA 1 ble con lesiones inflamatorias o similar". Como no existía una total evidencia de recidiva se decide seguimiento estricto del paciente con controles analíticos, radiológicos y manteniendo el tratamiento con Clorambucil. En enero de 2002 se practicó un nuevo TC en el que no se encontraron signos de recidiva.

Actualmente el paciente continua asintomático, con niveles estables de IgM (último control $48 \mathrm{~g} / \mathrm{l}$ ) a tratamiento con Clorambucil.

\section{DISCUSIÓN}

Los linfomas renales primarios son excepcionales, de forma que la afectación renal por un proceso linfoproliferativo es, por lo general, secundaria a una enfermedad sistémica ${ }^{1}$. Generalmente son los del tipo no-hodking los que presentan una mayor afectación extranodal, comprometiendo de forma más frecuente al tubo digestivo. En una serie de 621 pacientes con linfoma no-hodking a los que se practicó autopsia existía afectación de uno o ambos riñones en el $48 \%$ de los $\operatorname{casos}^{2}$. Habitualmente la afectación linfomatosa del riñón no suele plantear problemas de diagnóstico diferencial con otros tumores, ya que el linfoma renal se presenta habitualmente en pacientes con enfermedad diseminada como masas múltiples con aspecto característico en la ecografia. En la TC presentan una densidad homogénea, con realce homogéneo tras la administración de contraste y carácter infiltrativo ${ }^{3}$.

Sin embargo la presencia de un linfoma renal primario es un hallazgo poco frecuente, y controvertido desde el punto de vista fisiopatológico, ya que los riñones carecen de tejido linfático y por tanto el mecanismo de formación es poco claro ${ }^{4}$. Tampoco están bien definidos los criterios diagnósticos clínicos y radiológicos.

Clínicamente el proceso puede ser silencioso o provocar hematuria o una insuficiencia renal progresiva $^{5}$.

De forma general, el diagnóstico se basa en la ausencia de enfermedad extrarrenal. El TC resulta ser la prueba más sensible para la evaluación renal de pacientes con linfoma, en el que se presentan como lesiones isodensas en las exploraciones precontraste, que experimentan un menor realce que el parénquima normal tras la administración de contraste endovenoso al tratarse de 
lesiones escasamente vascularizadas. En RM son generalmente hipointensos respecto a la cortical renal en las secuencias T1 y ligeramente hipointensos a isointensos en $\mathrm{T}^{3}$.

El examen microscópico (Fig. 2) de este tipo de tumores demuestra un infiltrado difuso de células linfoides con núcleo oval y prominente nucleolo, moderadamente basófilos con diferenciación plasmocitoide, así como con numerosas mitosis ${ }^{6}$. Habitualmente son de tipo no-hodking, que son los que más afectación extranodal presentan. Nuestro caso fue descrito además como linfoma MALT (tumor linfoide asociado a las mucosas), que es una forma de linfoma extranodal asociada de forma frecuente a pacientes con linfoma gástrico Helicobacter Pylori positivo ${ }^{7}$.

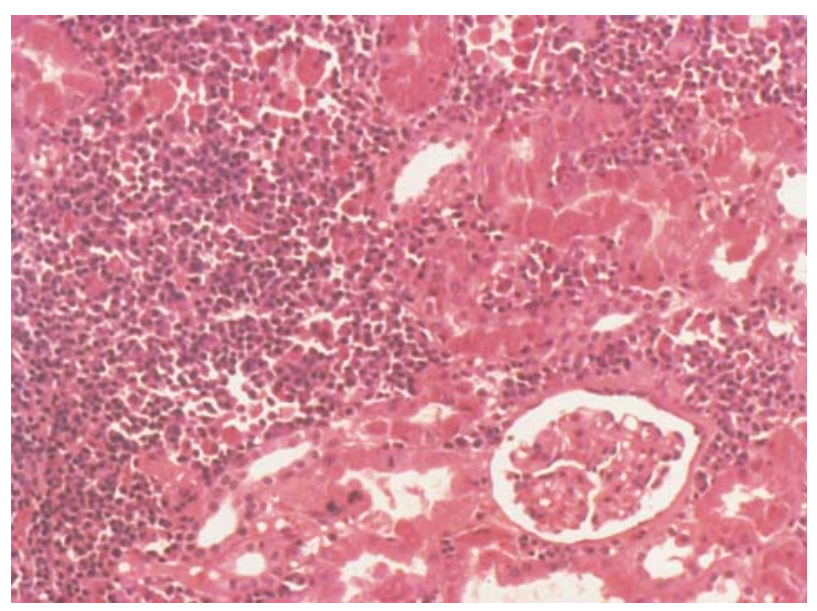

FIGURA 2

En cuanto al tratamiento sistémico, si existe afectación ganglionar, está contemplada la quimioterapia con ciclos de ciclofosfamida-doxorubicinavincristina-prednisolona (CHOP), asi como ifosfamida-etopósido-bleomicina (IMVP) ${ }^{8}$. En nuestro caso el paciente recibió solamente leukerán debido fundamentalmente a la gammapatía asociada.
Además de asociarse a gammapatía monoclonal como es nuestro caso, pueden debutar de forma bilateral con fracaso renal agudo ${ }^{8}$, hipercalcemia $^{9}$, pielonefritis xantogranulomatosa ${ }^{10} \mathrm{o}$ macroglobulinemia de waldestrom ${ }^{11}$.

\section{REFERENCIAS}

1. REQUENA MJ, LÓPEZ BELTRÁN A, ANGLADA FJ.: Neoplasias renales. Diagnóstico y tratamiento. Hospital Universitario Reina Sofía, 2001.

2. ARRANZ ARIJA JA, CARRIÓN R. J, GARCIA R. F y cols.: Primary renal lymphoma: report of 3 cases and review of the literature. American $J$ Nephrol 1994; 14: 148-153.

3. HERRANZ AMO F.: Tumores de riñón. Doyma, 2001.

4. GELLRICH J, HAKENBERG OW, NAUMANN R et al.: Primary renal non-hodgkin`s lymphoma. Onkologie 2002 jun; 25 (3): 273-277.

5. Campbell`S "Urology” 6 Edición; 2: 1082.

6. B. KANDEL L, KANDEL MD, DAVID L et al.: Primary renal lymphoma, does exist?. Cancer 1987; 60: 386-391.

7. HARRISON.: Principios de medicina interna. 14 Edición, compendio pg. 296.

8. CHOI JH, CHOI GB, SMI KN.: Bilateral primary non-hodkin's lymphoma presenting with acute renal failure: successful treatment with systemic chemotherapy. Acta Haematol 1997; 97 (4): 231235.

9. LEVENDOGLU-TUGAL O, KROOP S, ROZENBLIT GN et al. Primary renal lymphoma and hypercalcemia in a child leuk lymphoma 2002 may; 4 (5): 1141-1146.

10. KARADENIZ C, OGUZ A, ATAOGLU O et al.: Primary renal lynphoma and xantogranulomatous pyelonephritis in chilhood. J Nephrol 2002 sep-oct; 15 (5): 597-600.

11. CLIFTON AG, BAILY GG.: Primary renal lymphoma in a patient with waldenstrom's macroglobulinaemia. Clin Radiol 1991 apr; 43 (4): 285-286.

Dr. O. Rodríguez Faba

C/ Ámsterdam, 4 - $7^{\circ} \mathrm{A}$

33011 Oviedo (Asturias)

(Trabajo recibido el 4 marzo de 2003) 